This is the final peer-reviewed accepted manuscript of:

V. Kartsch, S. Benatti, M. Guermandi, F. Montagna and L. Benini, "Ultra Low-Power Drowsiness Detection System with BioWolf," 2019 9th International IEEE/EMBS Conference on Neural Engineering (NER), San Francisco, CA, USA, 2019, pp. 11871190.

The final published version is available online at: http://dx.doi.org/10.1109\%2FNER.2019.8717070

Rights / License:

The terms and conditions for the reuse of this version of the manuscript are specified in the publishing policy. For all terms of use and more information see the publisher's website. 


\title{
Ultra Low-Power Drowsiness Detection System with BioWolf
}

\author{
V. Kartsch*, S. Benatti*, M. Guermandi*, F. Montagna*, L.Benini ${ }^{* \ddagger}$ \\ ${ }^{*}$ DEI, University of Bologna, Italy. \\ Email: \{victorjavier.kartsch, simone.benatti, marco.guermandi, fabio.montagna, luca.benini\}@unibo.it \\ ${ }_{\ddagger}^{\ddagger}$ Integrated System Laboratory, ETHZ, Zurich, Switzerland. Email: lbenini@iis.ee.ethz.ch
}

\begin{abstract}
Drowsiness is a cause of accidents in industrial and mining activities. A considerable amount of effort has been put into the detection of drowsiness, and since then it has been integrated into a large variety of wearable systems. Nevertheless, the technology still suffers from high intrusiveness, short battery life and lack of generality. An opportunity to address these shortcomings arises from the use of physiological and behavioral features for bio-signals like EEG and IMU sensors. In this work, we propose an energy-efficient wearable platform for drowsiness detection. Our platform features a minimally invasive setup, based on dry EEG sensors to acquire neural data, and Mr. Wolf, an 8-core ultra-low-power digital platform. The system has been validated on three test subjects, achieving detection accuracy of $83 \%$, using a Nearest Centroid Classifier, modeled with a semi-supervised algorithm from previously collected data. This work further extends the capabilities of our previous system, providing a more sophisticated classification mechanism that includes real-time and onboard sensor fusion processing while running into a highly efficient and unobtrusive hardware platform, outperforming the current State of the Art (SoA) in terms of wearability and battery lifetime.
\end{abstract}

\section{INTRODUCTION}

One of the leading causes of accidents in material handling for the construction and mining industries can be ascribed to the mental state of the operator, mostly drowsiness and fatigue [1]. As operating machinery such as excavators, large trucks and cranes require a significant mental effort and stress, monitoring the operator attention level is a paramount objective in minimizing the risk of accidents. Thanks to recent developments in electronic systems and devices, several systems have been proposed to monitor attention levels. Most of them have been applied to the automotive sector where systems for automated advanced driving support are now available (e.g., lane assist, steering control, pedestrian protection), as well as systems for monitoring the driver attention (e.g., eye-trackers).

Systems capable of detecting drowsiness can be roughly divided into behavioral and physiological. The former analyzes behavioral patterns (e.g., eye blinking, nodding or body gestures) to determine the level of drowsiness while the latter continuously monitors one or a few physiological parameters for the same purpose. Behavioral systems make use of proxies to determine drowsiness, such as changes in the driving profile (e.g., skids, touching or crossing white lines) or in the driver's head gesture. Studies in [2] show how eye-blinking frequency and duration can be correlated to drowsiness. According to this, the duration is higher when the subject is heavy-eyed, being categorized as drowsy if the blinking duration is longer than $500 \mathrm{~ms}$. Similarly, blinking duration is used to detect micro-sleeps in [3]. Another significant parameter is named PERCLOS (proportion of time the eyes are at least $80 \%$ closed). The extraction of these parameters is usually done from data acquired with high-resolution cameras connected to the vehicle infotainment system. The main drawbacks of these computer-vision methods reside on the high-end expansive hardware required to process the images.
Moreover, these systems can work optimally only when the angle between the subject's eyes and the camera is inside predefined limits and if light conditions are good enough. These drawbacks make system portability almost impossible as the cameras are fixed and require careful tuning of the position [4].

To overcome these limitations, more direct approaches can be used, which target the monitoring of physiological parameters to determine the drowsiness level. It has also been demonstrated that these approaches significantly improve accuracy as well [5]. The main parameters whose use has been investigated so far are heart-rate, blood pressure or neural signals. Despite the fact that these systems can be designed to be wearable, the actual setups are too bulky to be considered socially acceptable for drivers and can therefore hardly be commercialized as consumer solutions. On the other hand, industrial applications can easily integrate wearable devices for drowsiness monitoring in mandatory personal protective equipment such as helmets and gloves.

Focusing on the analysis of neurological signals, different rythms can be separated from the raw EEG traces and used to determine drowsiness conditions, using machine learning algorithms [6]. However, inter-subject variability suggests that physiological analysis by itself is not sufficient to provide a strong enough generalization the drowsiness detection algorithms [7], and many proposed solutions rely on supervised and user-dependent approaches [8]. Furthermore, many of the aforementioned approaches are based on bench-top computational platforms, which are not suitable for wearable formfactored solutions. Keeping this in mind, this work, moving from the system developed in [9] introduces a fully wearable system capable of providing drowsiness alerts combining physiological and behavioral features analysis. The two major novels of the work are:

- the design and implementation of a wearable formfactored board (Biowolf) capable of acquiring up to 8 EEG channels and process the real-time neural data via a multicore chip (Mr. Wolf)

- the design of a semi-supervised learning algorithm based on $\mathrm{K}$-means clustering, that allows detecting drowsiness levels in a user-independent fashion,

By fusing information coming from the acquisition and processing of EEG signals (for monitoring of brain rhythms and eye blinking ) and an Inertial Measurement Unit (IMU, for monitoring head movements indicative of drowsiness, such as nodding), the proposed approach detects 4 levels of drowsiness, relying on the Nearest Centroid Classifier (NCC), a Machine Learning (ML) algorithm that offers a compelling trade-off between performance and computational power. The system is capable of detecting in real-time drowsiness levels with $83 \%$ accuracy, consuming only $6.1 \mathrm{~mW}$ and offering up to $60 \mathrm{~h}$ of autonomy. It can easily be integrated into safety protection devices like bump caps and helmets. 


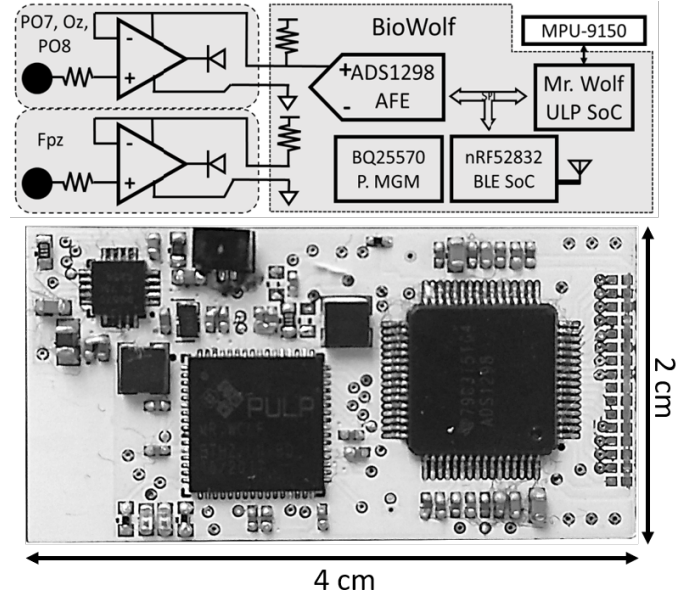

Fig. 1. PCB and hardware block diagram of BioWolf

\section{System SetuP}

The system presented in this work is based on the detection of behavioral and physiological indicators of drowsiness extracted from three EEG and IMU sensors. EOG artifacts on the EEG signals provide information about the blink duration that increases when drowsiness is present. On the other hand, the head gesture can also provide information about drowsiness. We extract these features and process them to ranks the drowsiness in four levels as indicated in Table I. The classification relies on the Nearest Centroid Classifier (NCC), a Machine Learning (ML) algorithm trained using k-Means, an unsupervised clustering method, from data collected experimentally of three test subjects, while meeting the drowsiness parameter criteria for each level, as reported in [9]. The entire classification is performed online on the Biowolf platform.

In this section, we present details of the system, including the feature extraction techniques, the training and classification, and the deployment on BioWolf.

\section{A. Embedded Architecture and Algorithm Implementation}

BioWolf is a highly-configurable platform for acquisition and embedded processing of biopotentials, supporting up to 8 differential channels. It is based on a multichannel commercial Analog Front End (AFE) and the Mr. Wolf SoC [10]. It also integrates a Nordic nRF52832 SoC for energy-efficient BLE connectivity to external devices and a Texas Instruments BQ25570 power management IC for ultralow power conversion and energy harvesting. The application makes use of three active EEG dry-electrodes placed according to the standard 10-20 system in PO7, Oz and PO8. The common reference electrode is placed in Fpz. Locations are chosen to maximize SNR in the acquisition of both relevant brain activity rhythms and eye blinking as needed by the application and to be compatible with integration on head caps, helmets and, headbands. The contact with the subject skin is obtained through commercial dry-contacts (g.SAHARA from g.tec Gmbh). Signal buffering is performed on the electrode by a low-power, low-noise, rail-to-rail Operational Amplifier (O.A., AD8603 from Analog Devices) connected in a noninverting configuration. This allows maximizing the SNR and resilience to interference even when contact quality is low, a typical occurrence in non-clinical settings. A protection resistor is used to limit patient current in case of system faults. To minimize the number of cables, the output signal of the amplification stage is used also for providing power supply to the O.A. with a forward-biased diode connecting the output pin of the O.A. to the power supply [11]. The output is biased by the following stage which entails a 10 $\mathrm{k} \Omega$ resistor toward a $3 \mathrm{~V}$ power supply.

The AFE is the de-facto standard used in biopotential acquisition platforms and presents a very favorable tradeoff between performance (especially noise level) and power consumption. As only 3 channels are used for the application, the other 5 are turned off to minimize power consumption.

Mr. Wolf SoC features eight near-threshold computing RISC-V programmable processors and up to $64+512 \mathrm{kB}$ of memory (L1 and L2, respectively), which provides the computational power to the embedded platform. The $\mathrm{SoC}$ also features a full set of peripherals managed by a multi-channel I/O DMA to minimize CPU utilization. The two SoCs and the AFE share the same SPI bus for data transfer.

An InvenSense MPU-9150 Motion Processing Unit (MPU) is used to capture the user motion. The MPU is a 9axis motion tracking device that combines a 3-axis MEMS gyroscope, a 3-axis MEMS accelerometer, 3-axis MEMS magnetometer and a Digital Motion Processor hardware accelerator. A $4 \times 2 \mathrm{~cm}$ four-layer Printed Circuit Board (PCB) assembles the described components. A block diagram and PCB layout of the complete hardware is presented in Fig. 1.

\section{B. Feature extraction of drowsiness indicators}

EEG data are acquired using a three-channel configuration. The first step of the preprocessing is to merge all the information contained in the channels using the Principal Component Analysis. PCA is a linear transformation which represents data into a new reference system by maximizing the variance contained in the original signals [12]. In this case, we reduce the dimensionality of the input matrix to a single vector, retaining up to $90 \%$ of the information. From this 1-D vector, we extract the features used for the drowsiness detection. Several features may reflect the state of drowsiness in EEG signals, but they are mostly related to the power in the lower bands of the EEG spectrum [13]. In this work, the first two features are based on the power of the signal in the alpha band (i.e. 8-13 Hz). The first, called Cumulative Alpha Power (CAP) accounts for bursts of alpha waves, which are increasingly present as drowsiness raises [13]. CAP is calculated by computing the alpha-band power spectrum over a moving-average filter with a large size ( $n=2 k$, or 2 seconds).

The second feature, called Constant Alpha Waves (CAW), aims to detect the closure of the eyes that is characterized by a constant presence of alpha waves with a relative large amplitude $(\tilde{1} 00 \mu \mathrm{V})$ and it is extracted by evaluating the signal power on the alpha band over a shorter movingaverage window ( $\mathrm{n}=500$ or $500 \mathrm{~ms})$. In Fig. 3, an example of the Alpha Waves power signal behavior is presented, which occurs when a subject closes the eyes.

TABLE I

SYSTEM DROWSINESS LEVELS

\begin{tabular}{cll}
\hline \multicolumn{2}{c}{ Levels of alarms with meeting criteria } \\
\hline Level & Description & Parameters \\
- & Fully awake subject (morning test) & $\mathrm{BD}<0.5 \mathrm{~s} \& \mathrm{AW}<\mathrm{Th} 1^{a}$ \\
1 & Increase of the blink duration & $\mathrm{BD}>0.5 \mathrm{~s}$ \\
2 & Increase of the alpha wave activity & $\mathrm{AW}>\mathrm{Th} 1^{a}$ \\
3 & Head gesture & $\mathrm{IMU}$ \\
4 & Closure of eyes & $\mathrm{AW}>\mathrm{Th} 2^{b}$ \\
\hline
\end{tabular}

Th1 ${ }^{a}$ AW over given threshold over a time window of 20 seconds. Th $2^{b}$ AW over given threshold over a time window of 3 seconds.

Data samples were stored after reaching some drowsiness criteria (previously acknowledged [9]) for each level. Physiological parameters were evoked by exposing the subject to drowsiness conditions or simulation. 


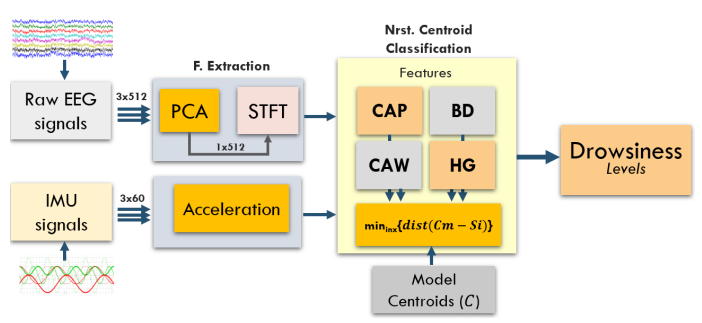

Fig. 2. Block diagram of the feature extraction and classification

The third feature, namely Blink Duration (BD) is also extracted from the EEG signal trace but it derives from an artifact related to an extra-ocular muscles movements. BD increases under drowsy conditions [2] and can be measured by exploiting the EOG artifacts introduced in the EEG signals. Fig. 4 shows the time-domain EEG signal (blue) and the power associated with the $1 \mathrm{~Hz}$ component (red). BD is estimated by calculating the time difference between a high and low peak (generated in correspondence with the blink duration) of the resulting signal.

The final feature is obtained by processing data from the IMU sensor, in particular, we compute the magnitude of the acceleration vector form the three cardinal coordinates, smoothed out using a moving average window $(n=1000)$. The output values are used to assess the movement of the head, where a low value characterizes a quietness, a high value represents sudden gestures (like a sudden tilt of the head), both present in drowsy conditions. Fig. 5 shows an example of the feature output signal including four sudden tilts.

\section{Semi-supervised training and classification}

The classification is based on the Nearest Centroid Classifier, where the output will depend on the distance from the average center of the labeled data (centroids). The learning process relies on finding the positions of such centroids, and it is performed automatically using the k-Means algorithm. K-Means clusters data by an iterative process where it first assigns a class label to all observations, as a function of the distance from the current class centroids and then reassigns such centroids to a new position in function of the average position of the recently labeled data. We run this on experimental data from three subjects simulating four different drowsy conditions $(k=4+1)$.

The initial positions of the centroids play a crucial role in the clustering. We compared two methods, the first one based on random assignment, the second one providing a portion of labeled data for fixing the initial centroid positions, which has been demonstrated to be helpful in increasing the robustness of this approach [14]. This is confirmed by our results presented in section III. Fig. 2 summarizes the process of feature extraction and classification.

Our proposed algorithm for the detection of the four levels of drowsiness was implemented taking advantage of

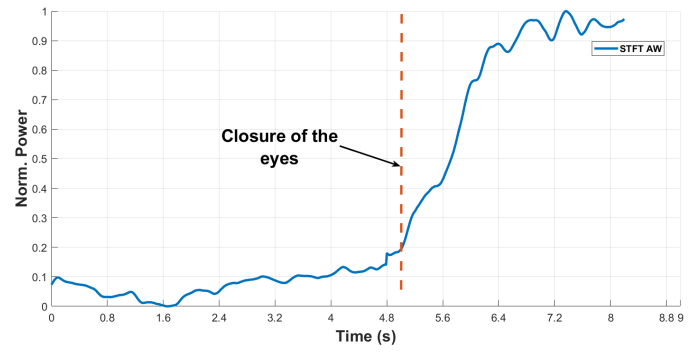

Fig. 3. Average normalized signal power in the Alpha band (8-13 Hz) computed on moving windows of $500 \mathrm{~ms}$

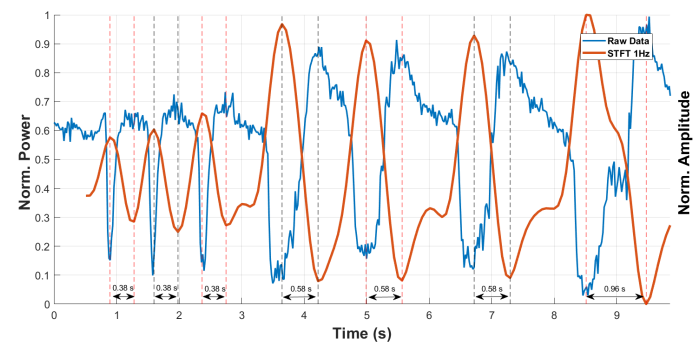

Fig. 4. Blinks in time/frequency domain. In blue, the original EEG signal with blinks artifacts. In red, 1-Hz signal energy, extracted using the STFT

the parallel capabilities of the hardware. The parallelization was performed using the OpenMP directives to distribute the workload of the kernels among the cores. Some of the PCA kernels are mostly executed sequentially due to iteration dependencies and workload unbalance, while the remaining are executed using four cores due to the small quantity of data. On the other hand, the FFT is fully parallelizable. Based on a radix-8 algorithm, each FFT butterfly performs a single Discrete Fourier Transform among eight samples in three stages. In this case, the speed-up performance is only limited by the number of FPUs (2) available on the MCU.

\section{EXPERIMENTAL RESULTS}

Complete testing of a drowsiness detection system is a complex task, which requires to be performed in a realcase scenario, possibly exposing test subjects to dangerous conditions, and it is therefore outside the scope of this paper. While simulators could provide a similar experience, it is acknowledged that the reactions of the subjects may not be trustful due to the awareness of being in a safe environment or distractions created by the experiment itself [18]. On the other hand, general guidelines for drowsiness detection have been introduced in previous investigations [19] and we can, therefore, rely on these results. The sampling and labeling of our datasets has been performed by carefully checking that drowsiness criteria are satisfied by the subject for each level, as indicated in Table I.

\section{A. Classification performance}

As introduced before, the NCC classification depends on the distance of the observation with respect to the class centroids, which contains information about the model of the classifier. K-means is used to determine the centroids' positions from the data collected from three test subjects simulating four different drowsiness levels. Nevertheless, the model can perform adversely if the initial conditions are not chosen adequately during the training phase. Using twofold cross-validation, we test two different training strategies. The first trains the model starting from randomly-initialized centroids, while in the second we provide a certain amount of labeled data to help the clustering. Table III demonstrates that the second approach leads to significant advantages. This is further confirmed by the online testing results reported in

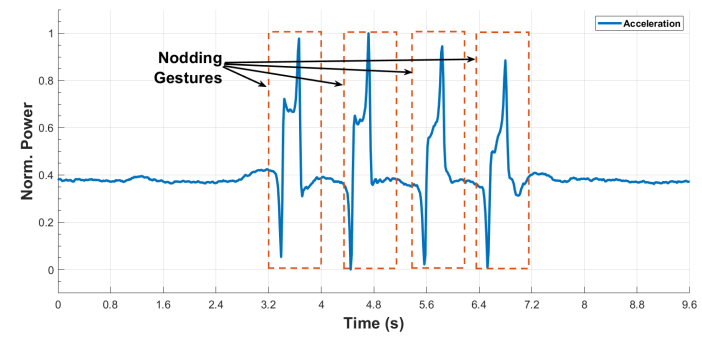

Fig. 5. Four nodding gestures extracted from the IMU sensors 
TABLE II

COMPARISON BETWEEN THE DIFFERENT SOA SYSTEMS AND THE CURRENT IMPLEMENTATION

\begin{tabular}{|c|c|c|c|c|c|c|}
\hline Author & Method & Advantage & Disadvantage & Accuracy[\%] & Platform & Intrusive \\
\hline$[15]$ & EOG, NNA & Online & Single feature & 87 & Embedded & NO \\
\hline$[6]$ & EEG, Neural Network & Single EEG Channel & Training, Offline & 83 & L. Computer & - \\
\hline$[16]$ & Camera, AdaBoost classifier & No training & Single feature & 94 & Emb. Computer & YES \\
\hline$[17]$ & NIRS, EEG, Alpha Pw & Portable & Noise, Redundant Measurements & $65-88$ & Microcontroller & NO \\
\hline This Work & EEG, IMU & Mult. features, Low-power & Noise & 83 & Microcontroller & NO \\
\hline
\end{tabular}

Table IV, showing an average accuracy of $83 \%$, with L3 and L4 above $90 \%$, ensuring the detection of the most critical drowsiness states.

\section{B. Power consumption}

As the majority of the wearable systems, an important aspect to take into account is the battery duration. In our system, the total power of the device is around $6.17 \mathrm{~mW}$, which is a contribution of the three main chips mounted on the board. The AFE is responsible for $36 \%$ of the power whether the Nordic MCU employs 43\%. The remaining power consumption derives from Mr. Wolf, and it is the result of the parallelization, the optimizations, and several power management techniques. Each $8 \mathrm{~ms}$ a new window of data is elaborated ( 8 samples overlap). The cluster elaborates the entire processing chain in less than $1 \mathrm{~ms}$ working with an operative frequency of $100 \mathrm{MHz}$ at $0.8 \mathrm{~V}$. During the processing, only the required cores of the cluster are clocked up avoiding energy loss. When the MCU is in idle, we power off the cluster and part of the SoC (sleep mode) to further reduce the power consumption. As a result, our system delivers up to $60 \mathrm{~h}$ of autonomy with a $200 \mathrm{mAh}$ battery, which can be further extended using the energy harvester subsystem. The resulting system exceeds the autonomy of current SoA systems [17], while providing an unobtrusive and fully wearable device for the detection of the drowsiness.

\section{Comparison with SoA}

Table II presents a comparison between the current drowsiness detection devices and the one presented in this work. Newer implementations make use of bench-top PCs to evaluate the drowsiness indicators since they require more computational effort, like in [6] where a Neural Network is used to detect the drowsiness. Our proposed implementation leverages a nimbler algorithm while exploiting behavioral and physiological parameters from many sources, thus, increasing the robustness of the detection. Indeed, systems in [15] and [16] only achieve higher accuracy on a single drowsiness parameter, hence they lack multi-parametric robustness. Moreover, the BD feature can estimate blink parameters with the same accuracy as camera-based systems like [16], while offering portability and unobtrusiveness at a much lower power budget. Our current system can be easily integrated into safety devices used in industry and mining activities, extending the battery life much further than other already available systems [17].

Nevertheless, our system can benefit from further improvements regarding accuracy, number of features, sensor fusion, and classification techniques. Also, advanced noise filtering can be adopted to provide robustness to EEG signals. As a matter of fact, the CPU utilization of the current application

TABLE III

K-MEANS OFFLINE TEST PER LEVEL

\begin{tabular}{|c|c|c|c|c|c|c|}
\hline $\begin{array}{l}\text { Method } \\
\text { Random } \\
\text { Pre-labeled }\end{array}$ & $\begin{array}{r}\mathbf{L O} \\
0.54 \\
0.73 \\
\end{array}$ & $\begin{array}{r}\text { L1 } \\
0.65 \\
0.71\end{array}$ & $\begin{array}{r}\mathbf{L 2} \\
0.40 \\
0.71\end{array}$ & $\begin{array}{r}\mathbf{L 3} \\
0.85 \\
1.00\end{array}$ & $\begin{array}{r}\mathbf{L 4} \\
0.95 \\
1.00\end{array}$ & $\begin{array}{l}\text { Avg } \\
0.67 \\
0.83\end{array}$ \\
\hline \multicolumn{7}{|c|}{$\begin{array}{c}\text { TABLE IV } \\
\text { ONLINE ACCURACY PER LEVEL }\end{array}$} \\
\hline $\begin{array}{l}\text { Method } \\
\text { T1 } \\
\text { T2 } \\
\text { T3 }\end{array}$ & $\begin{array}{r}\text { L0 } \\
0.83 \\
0.79 \\
0.82\end{array}$ & $\begin{array}{r}\text { L1 } \\
0.72 \\
0.75 \\
0.74\end{array}$ & $\begin{array}{r}\text { L2 } \\
0.74 \\
0.64 \\
0.70\end{array}$ & $\begin{array}{r}\mathbf{L 3} \\
1.00 \\
0.95 \\
0.96\end{array}$ & $\begin{array}{r}\mathbf{L 4} \\
0.95 \\
0.93 \\
0.94\end{array}$ & $\begin{array}{l}\text { Avg } \\
0.84 \\
0.81 \\
0.83\end{array}$ \\
\hline$\overline{A v g}$ & 0.81 & 0.74 & 0.69 & 0.97 & 0.94 & 0.83 \\
\hline
\end{tabular}

on Mr. Wolf is extremely low $(<10 \%)$, leaving plenty of room for more advanced computational analysis. Similarly, the system could be scaled to body-worn fashion by making use of in-ear/out-the-ear electrodes.

\section{CONCLUSIONS}

This work presents a drowsiness detection system featuring BioWolf, a Parallel Ultra Low Power platform, for biosignal processing that allows on-board, online and real-time classification. The system provides a high degree of wearability and embedded capabilities that can be easily integrated into safety protection devices. Our system, as demonstrated in the previous chapters, is in line with current SoA systems while consuming only $6.1 \mathrm{~mW}$, allowing a significant boost in battery life that can be further extended using the energy harvesting subsystem of BioWolf. This work further increases the capabilities of our previous system being more efficient, less intrusive, paving with it the way for a complete and universal wearable solution for drowsiness detection.

\section{ACKNOWLEDGMENT}

The research contribution presented has been supported by the European project EuroCPS (grant n. 644090).

\section{REFERENCES}

[1] J. M. Patterson et al., "Operator error and system deficiencies: analysis of 508 mining incidents and accidents from queensland, australia using hfacs," Accident Analysis \& Prevention, vol. 42, 2010.

[2] M. Ingre et al., "Subjective sleepiness, simulated driving performance and blink duration: examining individual differences," Journal of sleep research, 2006.

[3] G. Borghini et al., "Measuring neurophysiological signals in aircraft pilots and car drivers for the assessment of mental workload, fatigue and drowsiness," Neuroscience Biobehavioral Reviews, 2014.

[4] M. Flores, et al., "Driver drowsiness warning system using visual information for both diurnal and nocturnal illumination conditions," EURASIP, vol. 2010, no. 1, p. 3, 2010.

[5] A. Sahayadhas et al., "Detecting driver drowsiness based on sensors: A review," Sensors, vol. 12, no. 12, p. 16937, 2012.

[6] A. G. Correa et al., "Automatic detection of drowsiness in eeg records based on multimodal analysis," Medical Engineering Physics, 2014.

[7] B. H. Friedman et al., "Autonomic balance revisited: panic anxiety and heart rate variability," Journal of psychosomatic research, 1998.

[8] M. V. Yeo et al., "Can svm be used for automatic eeg detection of drowsiness during car driving?" Safety Science, 2009.

[9] V. Kartsch et al., "A wearable eeg-based drowsiness detection system with blink duration and alpha waves analysis," in NER, 2017.

[10] D. Rossi et al., "Energy-efficient near-threshold parallel computing: The pulpv2 cluster," IEEE Micro, vol. 37, no. 5, pp. 20-31, Sep 2017.

[11] M. Chiesi et al., "Creamino: a cost-effective, open-source eeg-based bci system," IEEE Transactions on Biomedical Engineering, 2018.

[12] F. Montagna et al., "Flexible, scalable and energy efficient bio-signals processing on the pulp platform: A case study on seizure detection," Journal of Low Power Electronics and Appl., 2017.

[13] H. J. Eoh, M. K. Chung, and S.-H. Kim, "Electroencephalographic study of drowsiness in simulated driving with sleep deprivation," International Journal of Industrial Ergonomics, 2005.

[14] S. Basu, A. Banerjee, and R. Mooney, "Semi-supervised clustering by seeding," in In Proceedings of 19th International Conference on Machine Learning (ICML-2002. Citeseer, 2002.

[15] K. M. Tabal and J. D. Cruz, "Development of low-cost embeddedbased electrooculogram blink pulse classifier for drowsiness detection system," in 2017 IEEE 13th International Colloquium on Signal Processing its Applications (CSPA), March 2017, pp. 29-34.

[16] J. W. Baek et al., "Real-time drowsiness detection algorithm for driver state monitoring systems," in 2018 Tenth International Conference on Ubiquitous and Future Networks (ICUFN), July 2018, pp. 73-75.

[17] U. Ha et al., "A multimodal drowsiness monitoring ear-module system with closed-loop real-time alarm," in BioCAS, Oct 2016.

[18] A. Sahayadhas et al., "Detecting driver drowsiness based on sensors: a review," Sensors, vol. 12, no. 12, pp. 16937-16953, 2012.

[19] M. E. Howard et al., "Specific sleepiness symptoms are indicators of performance impairment during sleep deprivation," Accident Analysis Prevention, vol. 62, pp. $1-8,2014$. 